\title{
Design and Construction of Link Quality and Localization Protocol Algorithms at WSN over IEEE802.15.4 Physical Protocol
}

\author{
Héctor Kaschel C. ${ }^{1}$, Luis Sánchez y B. ${ }^{2}$, José G. Mardones F. ${ }^{3}$, Gustavo Quezada C. ${ }^{3}$ \\ ${ }^{1}$ Departamento de Ingeniería Eléctrica, Facultad de Ingeniería, \\ hector.kaschel@usach.cl \\ 2 Departamento de Matemáticas, Facultad de Ciencias, \\ luis.sanchez@usach.cl \\ ${ }^{3}$ Doctorado en Ciencias de la Ingeniería, Mención Automática., \\ jose.mardonesf@usach.cl,gustavo.quezada@usach.cl.
}

\begin{abstract}
This work describes the steps for obtaining two link layer protocols for a wireless sensor network WSN, which functions integrated at single stack. The central axis for the development is the Received Signal Strength Indicator of the signal radio available at the implementation of the radio system in the IEEE802.15.4 standard. The protocols developed are quality link management protocols and positioning and localization protocols. The final results are completed with the protocols time synchronization, topology control and routing for generating a single protocols stack loading at nodes of WSN applied to industrial processes.
\end{abstract}

Keywords: Wireless Sensor Network, IEEE802.15.4, ZigBee, RSSI, Data Link Layer Protocol, Quality of Link, Link Indicator, Anchors, Positioning and Localization, Protocols Stack.

\section{Introduction}

Wireless Sensor Network WSN consists of individual nodes capable of interacting with their environment across sensing or physical parameters control; these nodes are collaborating amongst themselves to complete their tasks; tasks they cannot do individually. They use wireless communication for collaboration. In essence, the nodes are shaping a network because they have computer, wireless communication and control or sensing functions [1].

The origin of this work is the need to dispose of a WSN protocols stack whose performance can be considered as a complete object, fundamentally for three reasons:

- WSN will be applied in an industrial environment either as a plant or outdoor labor.

- If WSN is applied to this environment, two very important conditions will be presented: firstly, that the industrial processes will be functioning in real time and secondly, that the network is fault tolerant [1][2][3] to achieve a very high reliability rate.

- The current available hardware permits load software pieces of a size that allows each node to contain all the necessary information for building a protocols stack and has space for a host of other applications.
To meet with the given conditions, the software modules should be built as a single object with several options, for each protocol.

All network functionality is subordinated to the layer model, those sharing modules at the link and network layer, as shown in figure 1.

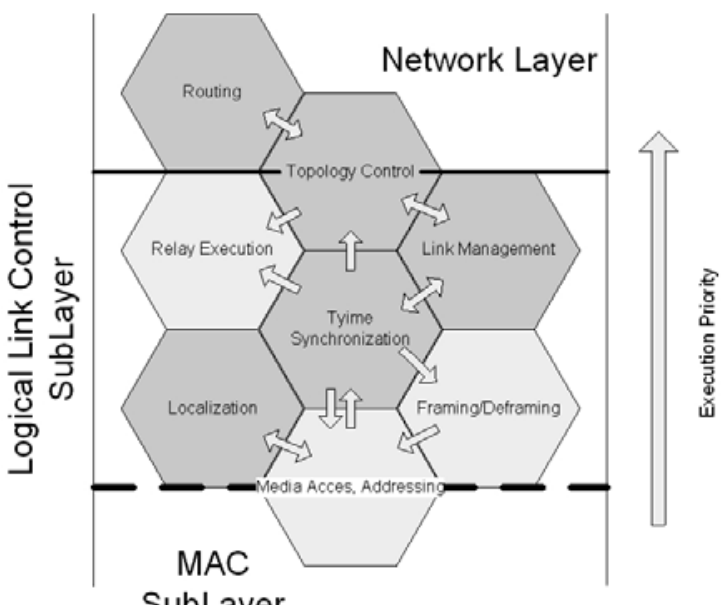

SubLayer

Figure 1. Protocols model by layer.

Figure 1 shows the implementation of link management and localization protocols that is necessary for the efficient performance of topology control and routing protocols, as the first step of development, which includes all mentioned protocols. In addition, the time synchronization protocol coordinates the execution of other protocols.

A review on current literature on this issue reveals no evidence of the development of an 
integral solution, as described in this paper, therefore this work is a new contribution to the field of WSN research.

Each software module contains a protocol that meets a specific function and is related to neighboring modules that share data and information. This allows a sequential machine to be simulated by parallel machine behavior.

In previous works the layer models are raised [4] to watch the phenomena from perspectives not necessarily compliant with the OSI model, or else focused on the data and service perspectives that the network supports.

Also, previous publications have defined protocols with similar functions but they work independently amongst themselves [5][6] without apparent intentions of generating a stack, as is proposed in this investigation. Also the treatment of the link management protocol is poor, especially when it is based on obtaining the Received Signal Strength Indicator (RSSI) parameter that offers a measurement tool for the power of received signals. There remains a doubt about the effectiveness since the errors produced at the lowest power (- $60 \mathrm{dBm}$ or less) require care with the treatment that the existing authors have been justified in using [7]. The author of the investigation produced evidence that validates the use of RSSI and contributes to the WSN layered protocols development.

The link management protocol directly affects other link layer protocols, as well as the network layer protocols, because by knowing the link quality one does not need to spend time and energy in framing, topology controlling and routing.

By respecting localization and positioning modules, there exist multiple proposals [1][8][9][10][11][12][13] y [14], that consider RSSI as a key factor for determining the distance between nodes that permit, by various methods, the setup position of each node into the network. Other methods include the Signal Angle of Reception and the Reception Differential of Time Arriving methods, but in both of these cases it is necessary to pay an additional cost, such as an addressable antenna for the first case and the dedicated clock algorithm which consumes machine cycles, for the second case.

The proposal developed here considers the RSSI, not from the neighboring nodes, but from anchors located at default positions that form an absolute coordinated system. There have been two attempts to develop this [ ][11], but neither attempt considered an absolute coordinated system. In one case a mobile anchor is used and in the other the information about the anchor's position is incorporated as data into packets that flow across the nodes. Therefore, evidence relative to these cases does exist.

With the intent to validate the hypothesis that the use of RSSI is the most reliable technique with the lowest cost, it is necessary prove in the field what some authors [7][8][16]have suggested through their own research: that that the RSSI at IEEE802.15.4 standard implementations, is between $-30 \mathrm{dBm}$ and -90 dBm [17]

To obtain a design according to what we want to achieve, a small network without load is built, in indoor and outdoor conditions and measurements of RSSI were obtained. This network works with nodes built over ZigBee technology, one node connected to a portable computer and another autonomous and mobile node, supplied by batteries.

The data collected through testing is considered as a reference and a justification for establishing a design criterion for an algorithm which would determine a quality link indicator. This would permit other protocols to take decisions of whether or not to do the work. If the decision is negative, then it would generate a timeout until the indicator is reliable.

The positioning and localization protocol is projected using the same RSSI to which applies a trilateralization algorithm. The RSSI was collected from three fixed anchors which mark the absolute coordinates. By computing the Euclidean distances algorithm, where the distances are obtained from the relationship between RSSI and the distance from the emitter, the approximated position into the coverage area from the network is determined.

When the network is operating fully, these protocols should work embedded into a microcontroller with a low memory and processing capacity, which is directly connected to a radio transceiver system ZigBee and the sensor: this set is called a sensor node.

This document is organized into sections; the Section II shows how to plan, organize and execute the testbed that permits the collection 
of the data, describing exactly the use of devices; later, in Section III the results of the measurements are shown, explaining the relationship with the scenarios in which they were obtained; later, section IV describes the aspects related to link management protocol development and how the quality indicator is deployed. Section V shows what, according to author, is a notable contribution to WSN research, such as the use of anchors or beacons in the construction of an absolute coordinated system, not only for localization processes but, also, for topology control. The Section VI specifies the development criteria and the algorithm for the localization and positioning protocols that use the RSSI parameter as the operational central axis, while the Section VII delivers the remaining challenges for future researches and the conclusions obtained..

\section{Testbed Implementation for Collecting Data.}

A configuration of nodes is organized as indicated below in Figure 2.
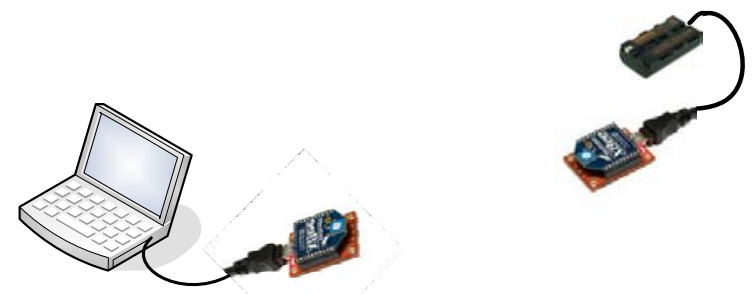

Figure 2. Implemented data capture testbed

Measurements were first taken in an open environment, or outdoors, and then in a closed environment, such as the inside of a building

The list of devices is shown in Table 1.

Table 1. Devices used in the testbed.

\begin{tabular}{|l|l|}
\hline \multicolumn{1}{|c|}{ Device } & \multicolumn{1}{|c|}{ Characteristics } \\
\hline $\begin{array}{l}\text { Módule Xbee 1 } \\
\mathrm{mW}\end{array}$ & $\begin{array}{l}\text { Receiver / Transmitter module with } \\
\text { antenna with chip based on Protocol } \\
\text { IEEE802.15.4, operating on 2.4 GHz } \\
\text { band. }\end{array}$ \\
\hline $\begin{array}{l}\text { XBee Explorer } \\
\text { Regulated }\end{array}$ & $\begin{array}{l}\text { Holding Platform with USB interface to } \\
\text { connect a data gatherer and energy } \\
\text { feeder through a USB port. }\end{array}$ \\
\hline USB Cord & $\begin{array}{l}\text { PC with USB port and executing X - } \\
\text { CTU software }\end{array}$ \\
\hline Laptop & $\begin{array}{l}\text { Base of support for XBee with battery } \\
\text { supply }\end{array}$ \\
\hline $\begin{array}{l}\text { Breakout Board } \\
\text { for } \\
\text { Module }\end{array}$ &
\end{tabular}

The portable PC, to which the Node XBee was connected, executed the software X-CTU, which, in turn, provided a screen with the gathered data. From this data, a reading of RSSI data was made which would later be used for research.

The obtained results were correlated to the measurements taken in an external environment or outdoors, and corresponding to those measurements taken inside a building or indoors.

The first measurement was taken at 11:30 hrs with partially cloudy skies and the avoidance of passing pedestrians, while the second measurement was taken at 16:45 hrs with very cloudy skies and cold temperatures. This measurement was taken at 12:00 hrs on an office building with normal personnel activity. Figure 3 shows the first measurement in outdoor conditions. In the graph depicting these measurements, it can be observed that as the nodes move away from each other the signal intensity starts to decrease.

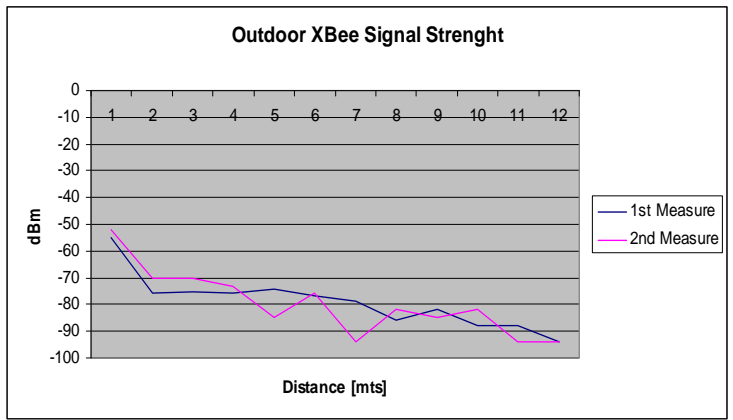

Figure 3. Graph for outdoor measurements surrounded by buildings

As other authors point out [4][5], the hardware signal intensity utilized is the same as indicated in the graphs shown in the previous figures. In an outdoor environment, behavior is more stable and predictable than in an indoor environment.

This can feasibly be confirmed by observing the graph shown in Figure 4.

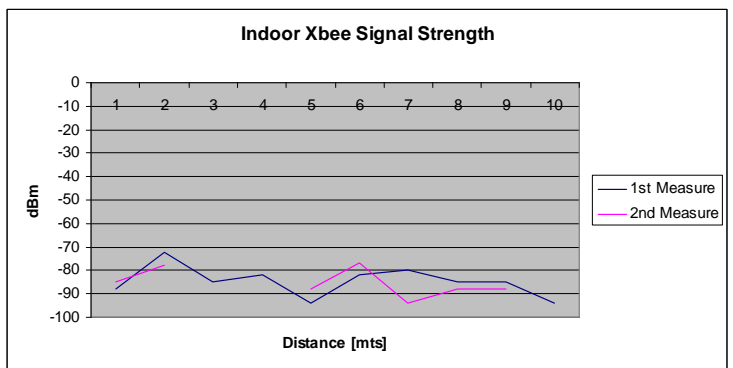

Figure 4. Graph of indoor measurements 
As it has been postulated by other authors [4][5], in an indoor environment the signal strength will be much more unstable fundamentally due to interferences caused by reflections off walls, ceilings and floors.

The second set of measurements was taken outdoors, on a plain area, without obstacles, within half an acre and this time with a greater frequency in the gathering of samples. The set also considers 49 measurements of distance and 150 measurements of signal power for each distance. This data is shown on the graph in Figure 5.

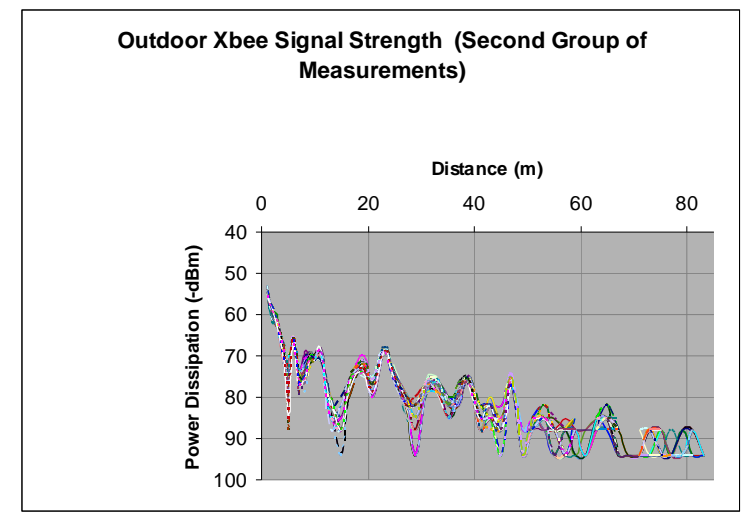

Figure 5. Graph for outdoor measurements on open ground.

In both cases of outdoor measurements (Figures 3 and 6), the graphs have similar trends, which are clearer in the graph shown in Figure 6. It shows an adjustment of the points to a model by the method of a logarithmic trend resulting in a negative exponential model, which absolutely coincides with the theoretical predictions.
Supported by empirical evidence, a concrete proposal can then be instigated with the view of implementing an application which may allow the acquisition of a link quality estimator that is based on the threshold values determined by the experimental results.

\section{Concepts Associated with Link Management Protocols and Criteria for Determining Quality Indicator and Generating Indicator Algorithm.}

The link management protocol is to compose a module from the link layer protocol, whose function is to collect reliable data of current state from the links with different neighbour nodes. These will be classified according to some criteria of stratification, and, depending on the results of this classification, interested protocols will be informed of whether they can or cannot use the links [7].

The criteria to be considered are:

- Link quality indicating the probability of losing packets over the link.[8]

- Link quality varies in time.

- Link quality must be estimated, either in an active way by the deliberate delivery of test packets or passively, by repetition of reception and evaluation of power transmission of neighbor nodes.[7]

The protocol must manage a link quality table of neighbor nodes which must be available for

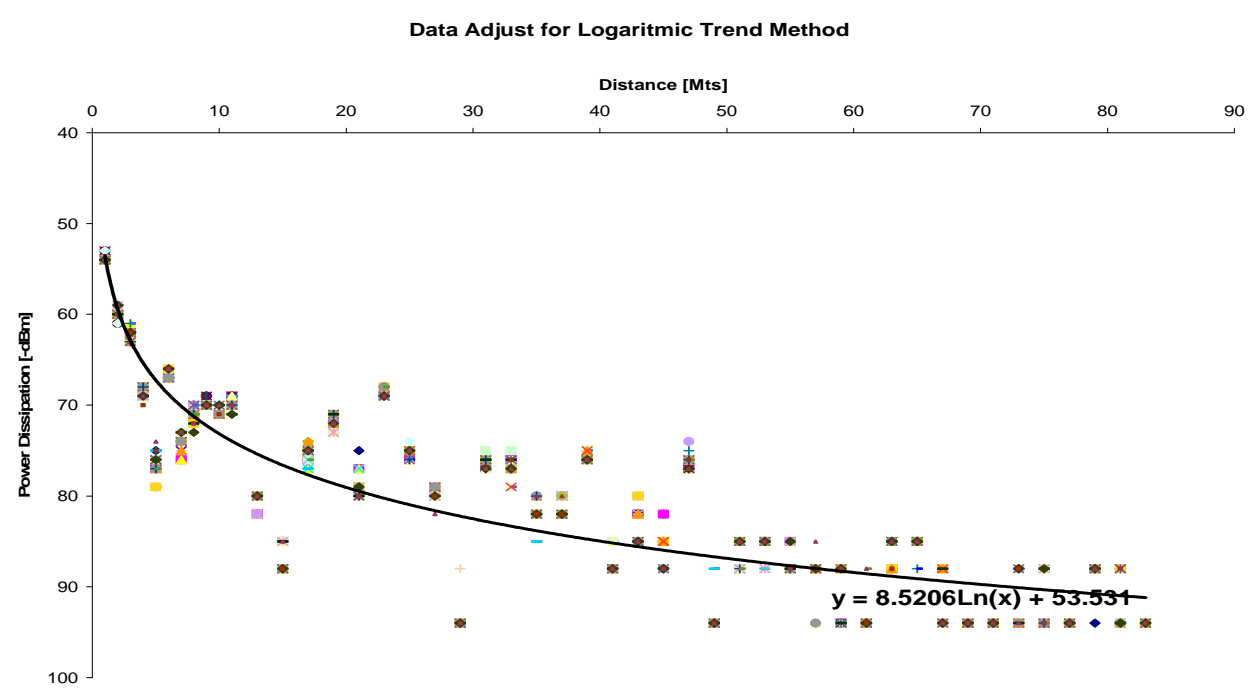

Figure 6. Graph for outdoor measurements with adjusted data. 
consultation by upper layer protocols or whoever needs this information.

Indicators can be active or passive. Active indicators use dedicated packets to fit the link, regardless of the traffic load with the corresponding delay and extra energy consumption, while the passive indicators use the same data of header data packets to obtain the indicator. The protocol IEEE802.15.4 and its implementation ZigBee, which was used during our work, allowed the transportation of the RSSI value which was recorded at a header field.

This field, called RSSI (Received Strength Signal Indicator) delivers a value at $-\mathrm{dBm}$, but is represented as a hexadecimal value. With the values from this field it is possible to sign in data from an algorithm denominated Quality Link Indicator Determination, which returns results as instantaneous quality link indicators.

The criteria for indicator determination must consider the functioning behaviour, both outdoor and indoor. But since the indoor behaviour is conclusively more erratic, it was decided that the values to be used for our model determination would be the outdoor data.

Power transformation from $\mathrm{dBm}$ to $\mathrm{mW}$ is given by the expression:

$$
d B m=10 \log \left(\frac{P_{r}}{1}\right)
$$

Using this expression an equivalence table between $\mathrm{dBm}$ and $\mathrm{mW}$ was built, for values less than $1 \mathrm{~mW}$, which is the range in which the results were presented when the test was made.

According to the vendor, the threshold value lower than the device Xbee received signal is $100 \mathrm{dBm}$, that correspond to $0.1 \mathrm{nW}$ [17].

For building the quality intervals, which generate the quality link indicator, three intervals were proposed, defined according to:

- BAD interval, where the RSSI values are less than $-80 \mathrm{dBm}$ or $10 \mathrm{nW}$.

- REGULAR interval, where the RSSI values are between $-80 \mathrm{dBm}$ and $-60 \mathrm{dBm}$ $(1 \mu \mathrm{W})$

- GOOD interval, where the RSSI values are greater than $-60 \mathrm{dBm}$.
Then the algorithm is presented.

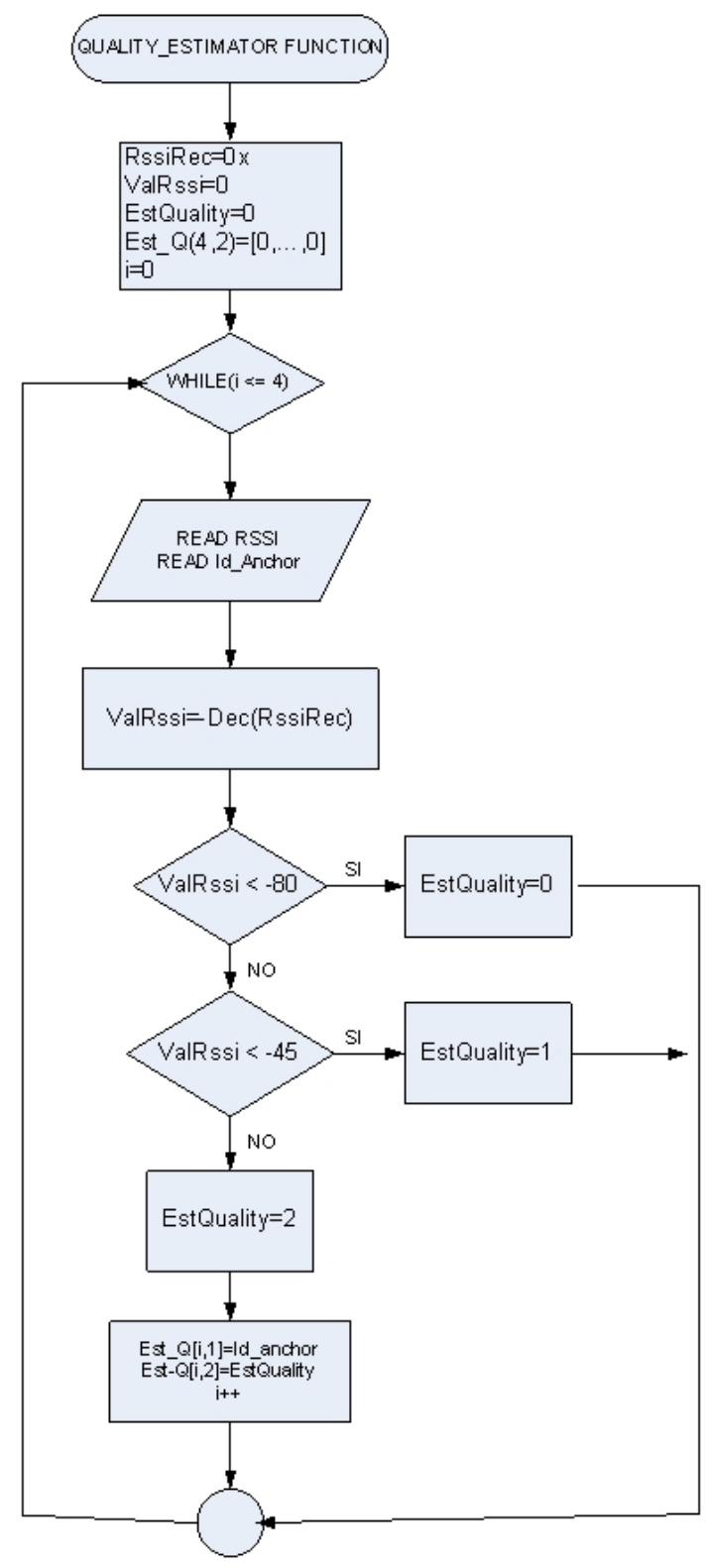

Figure 7. Quality Link Indicator is represented by 0,

1 y 2 values, meaning 0 as "BAD”, 1 as

"REGULAR" and 2 As "GOOD".

\section{Anchors Functionality to Support the Nodes Localization}

Anchors or beacons consist of an emitter node that sends a signal at same frequency channel as other nodes, but delivers an identifier data of an "only emitter" condition, such that every node can discriminate between this signal and that of others.[11]

The anchors are deployed at known fixed places and at coverage zone limits. Knowing the exact position of every anchor, they are set with a fixed and standard power level, so the 
RSSI evaluation by each node that receives the anchor signal will be the same, independent of which anchor is used (or has sent the signal).

This positioning strategy generates an absolute coordinate system that permits the calculation of the distances of nodes from a specific position.

Figure 8 shows a possible anchor location scheme which generates an absolute coordinate system that permits nodes to locate the anchors or beacons, registering the data as ordinate pair (RSSI, Anchor_Identificator).

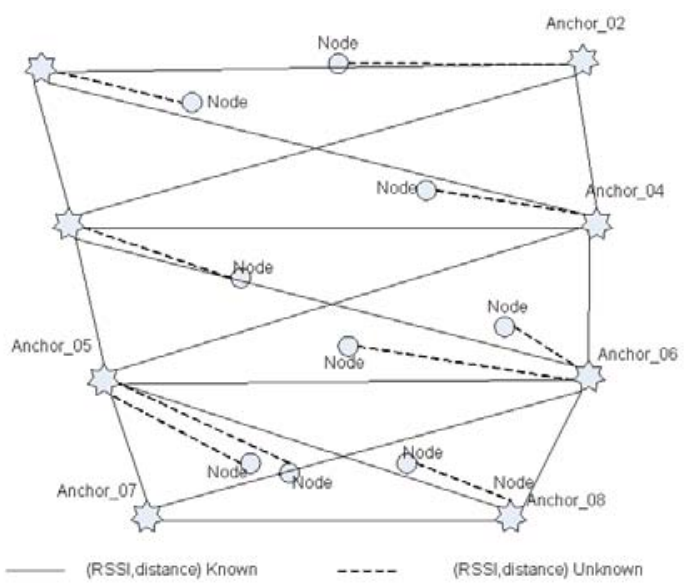

Figure 8. Anchorage schema at coverage zone of WSN.

Then the problem is determining the distance from the node to the nearest anchor. This evaluation is made by choice from the anchor ID from ordinate pairs that have the highest RSSI. Also, as an alternative method to be researched further, and knowing the data supplied by vendors and the radiation pattern from antennas of devices, it is possible to know the approximated angle of the anchor position with respect to the node that is doing the measurement. If both methods can be combined the algorithms available would be a more powerful method than those described by other researchers. Thus this is proposed for development with further research.

There is no evidence of another similar method currently under development.

\section{Localization and Positioning of Node Algorithm at WSN.}

The localization algorithm is built in five principal sections:

- Quality link indicator obtaining routine from every anchor.
- RSSI from each link selected routine

- Conversion RSSI to distance routine

- Construction and updating of anchor's table routine.

- Computation of localization by multilateralization method routine.

The first routine corresponding to the algorithm described in Section V determines the quality link indicator, in respect to one anchor only.

The second routine extracts the RSSI data corresponding to selected anchors.

The third routine converts RSSI in distance from the node to the anchor, using the formula:

$R S S I=-10 \log _{10} d+A$

Where A is the RSSI measured at 1 meter of distance below ideal conditions ${ }^{1}$ [1].

The fourth routine allows the obtained and computed data that every node contains to be inputted into a table. The table has the following fields; anchor, ID, RSSI, distance (in meters).

The fifth routine executes the multilateration process with all distances available to determine the exact node position and to deploy other protocols.

The algorithm is

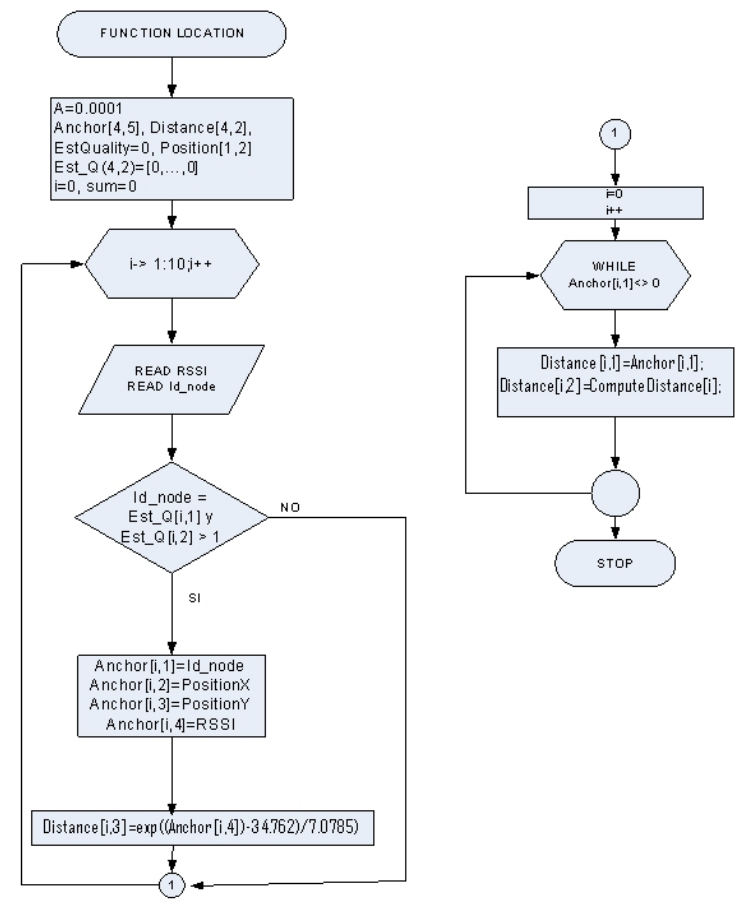

${ }^{1}$ Without interferences of another wireless network that works at same frequency band, outdoor, with green grass soil and to one meter high. 


\section{Future Line of Development and Conclusions.}

The immediate action required is to develop a programmed module built in at the sensor node, to verify the points made in this investigation, and so deploy the quality link indicator for use from other protocols, such as media access, localization and positioning, topology control and routing.

The quality link indicator is vital for the efficient performance of nodes at WSN. If the protocols dispose of the quality link indicator, they can save energy and decide which link offers the best conditions for transmission, providing efficiency to all networks.

It is possible to build a most elaborate procedure that permits a brief statistical analysis before generating the quality link indicator, avoiding the values that come associated with an error packet distorting the value of the indicator. This would generate a transmission doomed to failure.

Positioning and localization algorithm implementations are possible using the value of RSSI with a high level of reliability.

The use of anchors or beacons significantly favors a reduction in the complexity of the computing necessary for obtaining the position.

Moreover the cost of the RSSI and position coordinates is low. If it is not a method giving high accuracy level, then the reliability of the whole system will fall.

The methods used are considered fault tolerant, as three anchors are sufficient to do the computing operation of the position. But the condition that the system requires is a powerful link greater than $10 \mathrm{nW}$ for the anchor be considered reliable.

A future development line is the implementation of this solution over a network with hierarchical topology, cluster type, where the cluster head is located first and then it can help to locate the nodes belonging to its cluster.

Another pending task consists of comparing the obtained test results with the results obtained by some publications [1][6] and prove that the methods really offer an acceptable reliability.

The last pending task is to mix the multilateration method with the position estimation based on the RSSI to attain a more powerful method.

When these protocols are placed inside the nodes and the other modules have been developed, the resulting protocol stack will be a unique solution for WSN industrial application area.

\section{Acknowledgements}

The execution of this work was possible thanks to resources provided by the projects DICYT USACH Code 060933SYB "Desarrollo de una arquitectura de capas para distribuir funciones a sensores y actuadores, bajo criterios de confiabilidad y comunicación, en una Red Industrial de Sensores Inalámbricos” y DICYT USACH Code 060913KC "Diseño e implementación de una IWSN (Industrial Wireless Sensor Network) Tolerante e Fallas con Diversidad Cooperativa”.

\section{REFERENCES}

1. HOLGER, K., A. WILLIG, Protocols and Architectures for Wireless Sensor Networks. John Wiley \& Sons. 2005.

2. VITTURI, S., I. CARRERAS, D. MIORANDI, L. SCHENATO, A. SONA, Experimental Evaluation of an Industrial Application Layer Protocol over Wireless Systems. IEEE Transactions on Industrial Informatics, vol. 3, no. 4, November 2007, pp. 275-288.

3. CUCINOTTA, T., A. MANCINA, G. F. ANASTASI, G. LIPARI, L. MANGERUCA, R. CHECCOZZO, F. RUSINÀ, A Real-Time Service-Oriented Architecture for Industrial Automation. IEEE Transactions on Industrial Informatics, vol. 5, no. 3, August 2009. pp. 267-277.

4. NITAIGOUR, P. M., Sensor Networks and Configuration. Springer Science + Business Media B.V. 2007 ISBN-13 978-3540-37364-3.

5. WILLIG, A., M. KUBISCH, C. HOENE, A. WOLISZ, Measurements of a Wireless Link in an Industrial Environment using an IEEE 802.11-compliant Physical Layer., Transaction on Industrial Electronics, vol. 49, no. 6, December 2002, pp. 1265-1282. 
6. VERDONE, R., D. DARDARI, G. MAZZINI, A. CONTI, Wireless Sensor and Actuators Networks. Technologies, Analysis and Design. Academic Press. 2008. ISBN 978-0-12-372539-4.

7. SRINIVASAN, K., P. LEVI, RSSI is Under Appreciated, in Proceedings of the Third Workshop on Embedded Networked Sensors, 2006.

8. HAUER, J.-H., V. HANDZISKI, A. WOLISZ, Experimental Study of the Impact of WLAN Interference on IEEE 802.15.4 Body Area Networks Wireless Sensor Networks, 6th European Conference, EWSN 2009 Proceedings, Cork, Ireland, February 11-13, 2009, pp. 17-32.

9. PAPAMANTHOU, C., F. P. PREPARATA, R. TAMASSIA, Algorithms for Location Estimation Based on RSSI Sampling, Algorithmic Aspects of Wireless Sensor Networks. Fourth International Workshop ALGOSENSORS 2008 Reykjavik, Iceland, July 2008, Revised Selected Papers, pp. 72-86.

10. BOUKERCHE, A., H. A. B. F. OLIVEIRA, E. F. NAKAMURA, A. A. F. LOUREIRO, Localization Systems for Wireless Sensor Networks, Algorithms and Protocols for Wireless Sensor Networks, Azzedine Boukerche, PhD. John Wiley \& Sons, 2009, pp. 307-340.

11. KWON, T., J. H. LEE, J. S. SONG, Location-Based Pairwise Key Predistribution for Wireless Sensor Networks, IEEE Transactions on Wireless Communications, vol. 8, no. 11, November 2009, pp. 5436-5442.
12. SHUANG, T., Z. XIMMING, W. XINGUO, S. PENG, Z. HAIYANG, A Selective Anchor Node Localization Algorithm for Wireless Sensor Networks, 2007 International Conference on Convergence Information Technology Proceedings, pp. 358-362.

13. LAU, E.-E.-L., B.-G. LEE, S.-C. LEE, W.Y. CHUNG, Enhanced RSSI-based High Accuracy Real-Time User Location Tracking System for Indoor and Outdoor Environments, International Journal on Smart Sensing and Intelligent Systems, vol. 1, no. 2, June 2008, pp. 534548.

14. NIEWIADOMSKA-SZYNKIEWICZ, E., M. MICHAL, Optimization Schemes for Wireless Sensor Network Localization, International Journal of Applied Mathematics and Computer Science, vol. 19, no. 2, 2009, pp. 291-302.

15. HEIKO, W., N. DZIENGEL, J. SCHILLER, Distance-Based Distributed Multihop Localization in Mobile Wireless Sensor Networks, TechRepublic, September 2009.

16. ZHANG. Y. Z. B. F. Y. S. NING, A RSSI Based Localization Algorithm Using a Mobile Anchor Node for Wireless Sensor Networks, 2009 International Joint Conference on Computational Sciences and Optimization.

17. CASEY, P. R., K. E. TEPE, K. NARAYAN, Design and Implementation of a Testbed for IEEE 802.15.4 (Zigbee) Performance Measurements, EURASIP Journal on Wireless Communications and Networking, Volume 2010, 11 pages.

18. http://www.digi.com/products/wireless/zig bee-mesh/ 